\title{
EXISTENCE OF SOLUTIONS OF A CLASS OF ABSTRACT SECOND ORDER NONLINEAR INTEGRODIFFERENTIAL EQUATIONS
}

\author{
K. BALACHANDRAN \\ Bharathiar University \\ Department of Mathematics \\ Coimbatore 641 046, India \\ E-mail: balachandran_k@lycos.com \\ J.Y. PARK \\ Pusan National University \\ Department of Mathematics \\ Pusan 609-735, Korea \\ E-mail: jyepark@hyowon.pusan.ac.kr
}

(Received November, 2000; Revised September, 2001)

In this paper we prove the existence of solutions of nonlinear second order integrodifferential equations in Banach spaces. The results are obtained by using the theory of strongly continuous cosine families of operators and the Schaefer fixed point theorem.

Key words: Existence of Solutions, Second Order Integrodifferential Equation, Cosine Families of Operators, Schaefer's Theorem, Nonlocal Condition.

AMS subject classifications: $34 \mathrm{G} 20$.

\section{Introduction}

In this paper we shall consider the problem of existence of solutions of a special class of second order integrodifferential equations in Banach spaces. The physical origin of the problem lies in the theory of vibrations of an extendible beam of length $L$, with ends held a fixed distance apart, hinged or clamped, and is either stretched or compressed by an axial force, taking into account the fact that, during vibration, the elements of a beam perform not only a translatory motion, but also rotate $[5,14]$.

A mathematical model for this problem is an initial boundary value problem for the nonlinear hyperbolic equation

$$
\frac{\partial^{2} z}{\partial t^{2}}-\lambda \frac{\partial^{2} z}{\partial t^{2} \partial x^{2}}+\frac{\partial^{4} z}{\partial x^{4}}-\left(\alpha+\beta \int_{0}^{L}\left|\frac{\partial z(y t)}{\partial y}\right|^{2} d y\right) \frac{\partial^{2} z}{\partial x^{2}}=f\left(\frac{\partial z}{\partial t}\right),
$$

where $z(x, t)$ is the deflection of point $x$ at time $t, \alpha$ is a real constant proportional to the axial force acting on the beam when it is constrained to lie along the $x$ axis and $\lambda$ is a nonnegative constant. The nonlinearity of the equation is due to the extensibility of the beam. The above equation can be written in abstract form with the general nonlinear term as 


$$
(I-\lambda A) z^{\prime \prime}(t)+A^{2} z(t)-\left[\alpha+\beta M\left(\left\|A^{\frac{1}{2}} z\right\|^{2}\right)\right] A z(t)=f\left(t, z(t), z^{\prime}(t)\right)
$$

If we take $K=(I-\lambda A)$ and $\left[\alpha+\beta M\left(\left\|A^{\frac{1}{2}} z\right\|^{2}\right)\right] A-A^{2}=A$ for appropriate assumptions on $\alpha, \beta, M$, then equation (2) becomes

$$
K z^{\prime \prime}(t)=A z(t)+f\left(t, z(t), z^{\prime}(t)\right) .
$$

Hence, it is natural to consider the generalizations of the above equations as integrodifferential equations of the form

$$
K z^{\prime \prime}(t)=A z(t)+f\left(t, z(t), \int_{0}^{t} g\left(t, s, z(s), z^{\prime}(s)\right) d s, z^{\prime}(t)\right) .
$$

In many cases, it is advantageous to treat the second order abstract differential equations directly rather than to convert them into first order systems. A useful tool for the study of abstract second order equations is the theory of strongly continuous cosine families of operators $[15,16]$. The aim of this paper is to prove the existence of mild solutions in a Banach space with suitable conditions on the operators $K, A$ and the nonlinear functions $f$ and $g$. Related works to this kind of equation can be found in $[6,8,11,12]$.

\section{Preliminaries}

Consider the second order nonlinear integrodifferential equation of the form

$$
\begin{gathered}
K x^{\prime \prime}(t)=A x(t)+f\left(t, x(t), \int_{0}^{t} g\left(t, s, x(s), x^{\prime}(s)\right) d s, x^{\prime}(t)\right), t \in J=[0, T], \\
x(0)=x_{0} \in X, x^{\prime}(0)=y_{0} \in X
\end{gathered}
$$

where $K$ and $A$ are linear operators with domains contained in a Banach space $X$ and ranges contained in a Banach space $Y$, which satisfy certain conditions, and the nonlinear functions $g: J \times J \times X \times X \rightarrow X$ and $f: J \times X \times X \times X \rightarrow Y$ are given.

In order to prove our main theorem, we assume certain conditions on the operators $A$ and $K$. Let $X$ and $Y$ be Banach spaces with norm | · | and $\|\cdot\|$ respectively. The operators $A: D(A) \subset X \rightarrow Y$ and $K: D(K) \subset X \rightarrow Y$ satisfy the following hypothesis:

$\left(H_{1}\right) \quad A$ and $K$ are closed linear operators,

$\left(H_{2}\right) \quad D(K) \subset D(A)$ and $K$ is bijective,

$\left(H_{3}\right) \quad K^{-1}: Y \rightarrow D(K)$ is continuous.

Substituting $u(t)=K x(t)$ in equation (5) we get

$$
\begin{gathered}
u^{\prime \prime}(t)=A K^{-1} u(t) \\
+f\left(t, K^{-1} u(t), \int_{0}^{t} g\left(t, s, K^{-1} u(s),\left(K^{-1} u\right)^{\prime}(s)\right) d s,\left(K^{-1} u\right)^{\prime}(t)\right) .
\end{gathered}
$$

Now assume that

$\left(H_{4}\right) \quad A K^{-1}$ generates a strongly continuous cosine family $C(t), t \in R$, of bounded linear operators on $X$ and the adjoint operator $\left(A K^{-1}\right)^{*}$ is densely defined, that is, $\overline{D\left(\left(A K^{-1}\right)^{*}\right)}=X^{*}$ (see [2]).

We need the following fixed point theorem to prove our main result. 
Schaefer Theorem: [13] Let $E$ be a normed linear space. Let $F: E \rightarrow E$ be a completely continuous operator, and let

$$
\zeta(F)=\{x \in E: x=\lambda F x \text { for some } 0<\lambda<1\} .
$$

Then either $\zeta(F)$ is unbounded or $F$ has a fixed point.

Further, we make the following assumptions:

$\left(H_{5}\right) \quad C(t), t>0$ is compact.

$\left(H_{6}\right) \quad f(t, \cdot, \cdot, \cdot): X \times X \times X \rightarrow Y$ is continuous for each $t \in J$, and the function

$f(\cdot, x, y, z): J \rightarrow Y$ is strongly measurable for each $x, y, z \in X$.

$\left(H_{7}\right) \quad$ For every positive constant $k$ there exists $\alpha_{k} \in L^{1}(J)$ such that

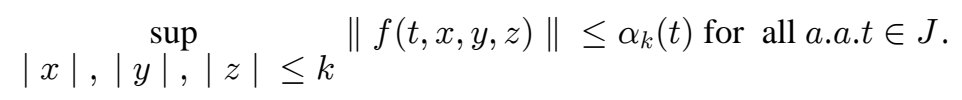

$\left(H_{8}\right) \quad g: J \times J \times X \times X \rightarrow X$ is continuous and there exist continuous functions $a, b: J \rightarrow[0, \infty)$ such that

$$
\|g(t, s, x, y)\| \leq a(t) b(s) \Omega_{0}(|x|+|y|), t, s \in J, x, y \in X,
$$

where $\Omega_{0}:[0, \infty) \rightarrow[0, \infty)$ is a continuous nondecreasing function.

$\left(H_{9}\right)$ There exists a continuous function $m: J \rightarrow[0, \infty)$ such that

$$
\|f(t, x, y, z)\| \leq m(t) \Omega(|x|+|y|+|z|), t \in J, x, y, z \in X,
$$

where $\Omega:[0, \infty) \rightarrow[0, \infty)$ is a continuous nondecreasing function.

For brevity, let us take

and

$$
\begin{gathered}
M=\sup \left\{\left\|K^{-1} C(t)\right\|: t \in J\right\}, M^{*}=\sup \left\{\left\|K^{-1} A K^{-1} S(t)\right\|: t \in J\right\}, \\
a_{0}=\sup \{a(t): 0 \leq t \leq T\}, \widetilde{m}(t)=\max \left\{(M+M T) m(t), a_{0} b(t)\right\}
\end{gathered}
$$

Also, assume that

$$
c=\left(M+M^{*}\right)\left\|K x_{0}\right\|+(M+M T)\left\|K y_{0}\right\| .
$$

$\left(H_{10}\right)$

$$
\int_{0}^{T} \tilde{m}(s) d s<\int_{c}^{\infty} \frac{d s}{\Omega(s)+\Omega_{0}(s)}
$$

It is well known that if $C(t)$ is a strongly continuous cosine family with infinitesimal generator $A K^{-1}$ and $x(t)$ is a solution of the problem (5)-(6), then

$$
\begin{gathered}
x(t)=K^{-1} C(t) K x_{0}+K^{-1} S(t) K y_{0} \\
+\int_{0}^{t} K^{-1} S(t-s) f\left(s, x(s), \int_{0}^{s} g\left(s, \tau, x(\tau), x^{\prime}(\tau)\right) d \tau, x^{\prime}(s)\right) d s .
\end{gathered}
$$

The above equation is more general than equation (5) and every solution of (8) is called a mild solution of (5)-(6). Further, equation (8) is easier to work with because of the nice properties of the bounded operators $C(t), S(t), t \in R$ as opposed to the unbounded operator $A K^{-1}$ in equation (7). 


\section{Main Result}

Theorem 3.1: Suppose $\left(H_{1}\right)-\left(H_{10}\right)$ hold. Then the problem (5)-(6) has at least one mild solution on $J$.

Proof: Consider the space $Z=C^{1}(J, X)$ with norm

$$
\|x\|^{*}=\max \left\{\|x\|,\left\|x^{\prime}\right\|\right\} .
$$

In order to study the existence problem for the equation (6)-(7), we apply the Schaefer theorem to the following equation as in [9]

$$
\begin{gathered}
K x^{\prime \prime}(t)=A x(t)+\lambda f\left(t, x(t), \int_{0}^{t} g\left(t, s, x(s), x^{\prime}(s)\right) d s, x^{\prime}(t)\right), t \in J, \\
x(0)=\lambda x_{0}, x^{\prime}(0)=\lambda y_{0}, \lambda \in(0,1) .
\end{gathered}
$$

Let $x$ be a mild solution of the problem (9)-(10). Then from

$$
\begin{gathered}
x(t)=\lambda\left(K^{-1} C(t) K x_{0}+K^{-1} S(t) K y_{0}\right) \\
+\lambda \int_{0}^{t} K^{-1} S(t-s) f\left(s, x(s), \int_{0}^{s} g\left(s, \tau, x(\tau), x^{\prime}(\tau)\right) d \tau, x^{\prime}(s)\right) d s
\end{gathered}
$$

we have

$$
\begin{gathered}
|x(t)| \leq M\left\|K x_{0}\right\|+M T\left\|K y_{0}\right\| \\
+M T \int_{0}^{t} m(s) \Omega\left(|x(s)|+\left|x^{\prime}(s)\right|+\int_{0}^{s} a(s) b(\tau) \Omega_{0}\left(|x(\tau)|+\left|x^{\prime}(\tau)\right|\right) d \tau\right) d s .
\end{gathered}
$$

Denoting by $v(t)$ the right-hand side of the above inequality, we have

$$
v(0)=M\left\|K x_{0}\right\|+M T\left\|K y_{0}\right\|,|x(t)| \leq v(t), t \in J,
$$

and

$$
\begin{aligned}
v^{\prime}(t)= & M T m(t) \Omega\left(|x(t)|+\left|x^{\prime}(t)\right|+\int_{0}^{t} a(t) b(s) \Omega_{0}\left(|x(s)|+\left|x^{\prime}(s)\right|\right) d s\right) \\
& \leq \operatorname{MTm}(t) \Omega\left(v(t)+\left|x^{\prime}(t)\right|+a_{0} \int_{0}^{t} b(s) \Omega_{0}\left(v(s)+\left|x^{\prime}(s)\right|\right) d s\right)
\end{aligned}
$$

Therefore, if $u(t)=\sup \left\{\left\|x^{\prime}(s)\right\|: s \in[0, t]\right\}, t \in J$, then

$$
v^{\prime}(t) \leq \operatorname{MTm}(t) \Omega\left(v(t)+u(t)+a_{0} \int_{0}^{t} b(s) \Omega_{0}(v(s)+u(s)) d s\right) .
$$

But

$$
x^{\prime}(t)=\lambda\left[K^{-1} A K^{-1} S(t) K x_{0}+K^{-1} C(t) K y_{0}\right]
$$




$$
+\lambda \int_{0}^{t} K^{-1} C(t-s) f\left(s, x(s), \int_{0}^{s} g\left(s, \tau, x(\tau), x^{\prime}(\tau)\right) d \tau, x^{\prime}(s)\right) d s .
$$

Thus we have,

$$
\begin{gathered}
u(t) \leq M^{*}\left\|K x_{0}\right\|+M\left\|K y_{0}\right\| \\
+M \int_{0}^{t} m(s) \Omega\left(v(s)+u(s)+\int_{0}^{s} a(s) b(\tau) \Omega_{0}(v(\tau)+u(\tau)) d \tau\right) d s .
\end{gathered}
$$

Denoting by $r(t)$ the right-hand side of the above inequality, we have

$$
u(t) \leq r(t), r(0)=M^{*}\left\|K x_{0}\right\|+M\left\|K y_{0}\right\|, t \in J
$$

and

$$
\begin{aligned}
& r^{\prime}(t)=M m(t) \Omega\left(v(t)+u(t)+\int_{0}^{t} a(t) b(s) \Omega_{0}(v(s)+u(s)) d s\right) \\
& \leq M m(t) \Omega\left(v(t)+r(t)+a_{0} \int_{0}^{t} b(s) \Omega_{0}(v(s)+r(s)) d s\right), t \in J .
\end{aligned}
$$

Let

$$
w(t)=v(t)+r(t)+a_{0} \int_{0}^{t} b(s) \Omega_{0}(v(s)+r(s)) d s .
$$

Then

$$
w(0)=v(0)+r(0)=c, v(t)+r(t) \leq w(t), t \in J,
$$

and

$$
\begin{gathered}
w^{\prime}(t)=v^{\prime}(t)+r^{\prime}(t)+a_{0} b(t) \Omega_{0}(v(t)+r(t)) \\
\leq(M+M T) m(t) \Omega(w(t))+a_{0} b(t) \Omega_{0}(w(t)) \\
=\widetilde{m}(t)\left[\Omega(w(t))+\Omega_{0}(w(t))\right], t \in J .
\end{gathered}
$$

This implies

$$
\int_{w(0)}^{w(t)} \frac{d s}{\Omega(s)+\Omega_{0}(s)} \leq \int_{0}^{T} \widetilde{m}(s) d s<\int_{c}^{\infty} \frac{d s}{\Omega(s)+\Omega_{0}(s)}, t \in J .
$$

This inequality implies that there is a constant $M_{0}$ such that

$$
w(t) \leq M_{0}, t \in J
$$

Then 


$$
\|x(t)\| \leq v(t), \quad\left\|x^{\prime}(t)\right\| \leq u(t) \leq r(t), t \in J,
$$

and hence

$$
\|x\|^{*}=\max \left\{\|x\|,\left\|x^{\prime}\right\|\right\} \leq M_{0},
$$

where $M_{0}$ depends only on $T$ and on the functions $m, a, b, \Omega_{0}$ and $\Omega$.

We shall now prove that the operator $F: Z \rightarrow Z$ defined by

$$
\begin{gathered}
(F x)(t)=K^{-1} C(t) K x_{0}+K^{-1} S(t) K y_{0} \\
+\int_{0}^{t} K^{-1} S(t-s) f\left(s, x(s), \int_{0}^{s} g\left(s, \tau, x(\tau), x^{\prime}(\tau)\right) d \tau, x^{\prime}(s)\right) d s
\end{gathered}
$$

is a completely continuous operator.

Let $B_{k}=\left\{x \in Z:\|x\|^{*} \leq k\right\}$ for some $k \geq 1$. We first show that $F$ maps $B_{k}$ into an equicontinuous family. Let $x \in B_{k}$ and $t_{1}, t_{2} \in J$. Then if $0<t_{1}<t_{2} \leq T$,

$$
\begin{gathered}
\left\|(F x)\left(t_{1}\right)-(F x)\left(t_{2}\right)\right\| \\
\leq \| K^{-1}\left[C\left(t_{1}\right)-C\left(t_{2}\right)\|\| K x_{0}\|+\| K^{-1}\left[S\left(t_{1}\right)-S\left(t_{2}\right)\right]\|\| K y_{0} \|\right. \\
+\| \int_{0}^{t_{1}} K^{-1}\left[S\left(t_{1}-s\right)-S\left(t_{2}-s\right)\right] f\left(s, x(s), \int_{0}^{s} g\left(s, \tau x(\tau), x^{\prime}(\tau)\right) d \tau, x^{\prime}(s)\right) d s \\
\quad+\int_{t_{1}}^{t_{2}} K^{-1} S\left(t_{2}-s\right) f\left(s, x(s), \int_{0}^{s} g\left(s, \tau, x(\tau), x^{\prime}(\tau)\right) d \tau, x^{\prime}(s)\right) d s \| \\
\leq\left\|K^{-1}\left[C\left(t_{1}\right)-C\left(t_{2}\right)\right]\right\|\left\|K x_{0}\right\|+\left\|K^{-1}\left[S\left(t_{1}\right)-S\left(t_{2}\right)\right]\right\|\left\|K y_{0}\right\| \\
+\int_{0}^{t_{1}}\left\|K^{-1}\left[S\left(t_{1}-s\right)-S\left(t_{2}-s\right)\right]\right\| \alpha_{k}(s) d s+M T \int_{t_{1}}^{t_{2}} \alpha_{k}(s) d s
\end{gathered}
$$

and similarly,

$$
\begin{gathered}
\left\|(F x)^{\prime}\left(t_{1}\right)-(F x)^{\prime}\left(t_{2}\right)\right\| \\
\leq \| K^{-1}\left[C^{\prime}\left(t_{1}\right)-C^{\prime}\left(t_{2}\right)\|\| K x_{0}\|+\| K^{-1}\left[S^{\prime}\left(t_{1}\right)-S^{\prime}\left(t_{2}\right)\right]\|\| K y_{0} \|\right. \\
+\int_{0}^{t_{1}}\left\|K^{-1}\left[C\left(t_{1}-s\right)-C\left(t_{2}-s\right)\right] f\left(s, x(s), \int_{0}^{s} g\left(s, \tau x(\tau), x^{\prime}(\tau)\right) d \tau, x^{\prime}(s)\right)\right\| d s \\
+\left\|\int_{t_{1}}^{t_{2}} K^{-1} C\left(t_{2}-s\right) f\left(s, x(s), \int_{0}^{s} g\left(s, \tau, x(\tau), x^{\prime}(\tau)\right) d \tau, x^{\prime}(s)\right) d s\right\| \\
\leq\left\|K^{-1} A K^{-1}\left(S\left(t_{1}\right)-S\left(t_{2}\right)\right)\right\|\left\|K x_{0}\right\|+\left\|K^{-1}\left[C\left(t_{1}\right)-C\left(t_{2}\right)\right]\right\|\left\|K y_{0}\right\| \\
+\int_{0}^{t_{1}}\left\|K^{-1}\left[C\left(t_{1}-s\right)-C\left(t_{2}-s\right)\right]\right\| \alpha_{k}(s) d s+M \int_{t_{1}}^{t_{2}} \alpha_{k}(s) d s .
\end{gathered}
$$


The right-hand sides are independent of $x \in B_{k}$ and tend to zero as $t_{1} \rightarrow t_{2}$, since $C(t)$, $S(t)$ are uniformly continuous for $t \in J$ and the compactness of $C(t), S(t)$ for $t>0$ implies the continuity in the uniform operator topology. The compactness of $S(t)$ follows from that of $C(t)$. Thus $F$ maps $B_{k}$ into an equicontinuous family of functions. It is easy to see that the family $F B_{k}$ is uniformly bounded.

Next we show $\overline{F B_{k}}$ is compact. Since we have shown $F B_{k}$ is an equicontinuous collection, it suffices by the Arzela-Ascoli theorem to show that $F$ maps $B_{k}$ into a precompact set in $X$.

Let $0<t \leq T$ be fixed and $\epsilon$ a real number satisfying $0<\epsilon<t$. For $x \in B_{k}$ we define

$$
\left(F_{\epsilon} x\right)(t)=K^{-1} C(t) K x_{0}+K^{-1} S(t) K y_{0}+\int_{0}^{t-\epsilon} K^{-1} S(t-s) f\left(s, x(s), x^{\prime}(s)\right) d s .
$$

Since $C(t), S(t)$ are compact operators, the set $Y_{\epsilon}(t)=\left\{\left(F_{\epsilon} x\right)(t): x \in B_{k}\right\}$ is precompact in $X$ for every $\epsilon, 0<\epsilon<t$. Moreover for every $x \in B_{k}$ we have

$$
\begin{gathered}
\left\|(F x)(t)-\left(F_{\epsilon} x\right)(t)\right\| \\
\leq \int_{t-\epsilon}^{t}\left\|K^{-1} S(t-s) f\left(s, x(s), \int_{0}^{s} g\left(s, \tau, x(\tau), x^{\prime}(\tau)\right) d \tau, x^{\prime}(s)\right)\right\| d s \\
\leq M T \int_{t-\epsilon}^{t} \alpha_{k}(s) d s
\end{gathered}
$$

and

$$
\begin{gathered}
\left\|(F x)^{\prime}(t)-\left(F_{\epsilon} x\right)^{\prime}(t)\right\| \\
\leq \int_{t-\epsilon}^{t}\left\|K^{-1} C(t-s) f\left(s, x(s), \int_{0}^{s} g\left(s, \tau, x(\tau), x^{\prime}(\tau)\right) d \tau, x^{\prime}(s)\right)\right\| d s \\
\leq M \int_{t-\epsilon}^{t} \alpha_{k}(s) d s .
\end{gathered}
$$

Therefore, there are precompact sets arbitrarily close to the set $\left\{(F x)(t): x \in B_{k}\right\}$. Hence, the set $\left\{(F x)(t): x \in B_{k}\right\}$ is precompact in $X$.

It remains to show that $F: Z \rightarrow Z$ is continuous. Let $\left\{x_{n}\right\}_{0}^{\infty} \subseteq Z$ with $x_{n} \rightarrow x$ in $Z$. Then there is an integer $q$ such that $\left\|x_{n}(t)\right\| \leq q$, $\left\|x_{n}^{\prime}(t)\right\| \leq q$ for all $n$ and $t \in J$, so $\|x(t)\| \leq q,\left\|x^{\prime}(t)\right\| \leq q$ and $x, x^{\prime} \in Z$. By $\left(H_{6}\right)$,

$$
f\left(t, x_{n}(t), \int_{0}^{t} g\left(t, s, x_{n}(s), x_{n}^{\prime}(s)\right) d s, x_{n}^{\prime}(t)\right) \rightarrow f\left(t, x(t), \int_{0}^{t} g\left(t, s, x(s), x^{\prime}(s)\right) d s, x^{\prime}(t)\right)
$$

for each $t \in J$ and since

$$
\begin{gathered}
\left\|f\left(t, x_{n}(t), \int_{0}^{t} g\left(t, s, x_{n}(s), x_{n}^{\prime}(s)\right) d s, x_{n}^{\prime}(t)\right)-f\left(t, x(t), \int_{0}^{t} g\left(t, s, x(s), x^{\prime}(s)\right) d s, x^{\prime}(t)\right)\right\| \\
\leq 2 \alpha_{q}(t)
\end{gathered}
$$


we have by the dominated convergence theorem

$$
\begin{aligned}
\left\|F x_{n}-F x\right\|= & \sup _{t \in J} \| \int_{0}^{t} K^{-1} S(t-s)\left[f\left(s, x_{n}(s), \int_{0}^{s} g\left(s, \tau, x_{n}(\tau), x_{n}^{\prime}(\tau)\right) d \tau, x_{n}^{\prime}(s)\right)\right. \\
& \left.\quad-f\left(s, x(s), \int_{0}^{s} g\left(s, \tau, x(\tau), x^{\prime}(\tau)\right) d \tau, x^{\prime}(s)\right)\right] d s \| \\
\leq & M T \int_{0}^{t} \| f\left(s, x_{n}(s), \int_{0}^{s} g\left(s, \tau, x_{n}(\tau), x_{n}^{\prime}(\tau)\right) d \tau, x_{n}^{\prime}(s)\right) \\
& -f\left(s, x(s), \int_{0}^{s} g\left(s, \tau, x(\tau), x^{\prime}(\tau)\right) d \tau, x^{\prime}(s)\right) \| d s \rightarrow 0
\end{aligned}
$$

and

$$
\begin{gathered}
\left\|\left(F x_{n}\right)^{\prime}-(F x)^{\prime}\right\| \\
=\sup _{t \in J} \| \int_{0}^{t} K^{-1} C(t-s)\left[f\left(s, x_{n}(s), \int_{0}^{s} g\left(s, \tau, x_{n}(\tau), x_{n}^{\prime}(\tau)\right) d \tau, x_{n}^{\prime}(s)\right)\right. \\
\left.-f\left(s, x(s), \int_{0}^{s} g\left(s, \tau, x(\tau), x^{\prime}(\tau)\right) d \tau, x^{\prime}(s)\right)\right] d s \| \\
\leq M \int_{0}^{t} \| f\left(s, x_{n}(s), \int_{0}^{s} g\left(s, \tau, x_{n}(\tau), x_{n}^{\prime}(\tau)\right) d \tau, x_{n}^{\prime}(s)\right) \\
-f\left(s, x(s), \int_{0}^{s} g\left(s, \tau, x(\tau), x^{\prime}(\tau)\right) d \tau, x^{\prime}(s)\right) \| d s \rightarrow 0, \text { as } n \rightarrow \infty .
\end{gathered}
$$

Thus $F$ is continuous. This completes the proof that $F$ is completely continuous.

Finally, the set $\zeta(F)=\{x \in Z: x=\lambda F x, \lambda \in(0,1)\}$ is bounded, as we proved in the first step. Consequently, by Schaefer's theorem the operator $F$ has a fixed point in $Z$. This means that any fixed point of $F$ is a mild solution of (5) on $J$ satisfying $(F x)(t)=x(t)$.

\section{Nonlocal Cauchy Problem}

In this section we shall consider the nonlocal Cauchy problem for the nonlinear second order integrodifferential equation (5). This problem was first studied by Byszewski [3] for semilinear equations in Banach spaces. Subsequently several authors have studied the same problem for various classes of differential and integrodifferential equations in Banach spaces $[1,4,7,10]$.

Consider the second order nonlinear integrodifferential equation with nonlocal condition of the form

$$
K x^{\prime \prime}(t)=A x(t)+f\left(t, x(t), \int_{0}^{t} g\left(t, s, x(s), x^{\prime}(s)\right) d s, x^{\prime}(t)\right), t \in J,
$$




$$
x(0)+h(x)=x_{0}, x^{\prime}(0)=y_{0},
$$

where $K, A, g, f$ are as before and $h: C(J, X) \rightarrow X$ is uniformly bounded.

By the hypothesis $\left(H_{1}\right)-\left(H_{4}\right)$, the solution of equation (11)-(12) is

$$
\begin{gathered}
x(t)=K^{-1} C(t) K\left[x_{0}-h(x)\right]+K^{-1} S(t) K y_{0} \\
+\int_{0}^{t} K^{-1} S(t-s) f\left(s, x(s), \int_{0}^{s} g\left(s, \tau, x(\tau), x^{\prime}(\tau)\right) d \tau, x^{\prime}(s)\right) d s .
\end{gathered}
$$

For nonlocal term $h$, we assume the following condition.

$\left(H_{11}\right) \quad h: C(J ; X) \rightarrow X$ and there exists a constant $G>0$ such that

$$
\|h(x)\| \leq G, \text { for } x \in C(J ; X)
$$

and the set $\left\{x(0): x \in C(J ; X),|x| \leq k, x(0)=x_{0}-h(x)\right\}$ is precompact in $X$.

Then the existence of mild solutions for the problem (11)-(12) can be established in a similar way as in Theorem 3.1 where the constant $c$ is taken as $c=\left(M+M^{*}\right)$ $\left(\left\|K x_{0}\right\|+G\right)+(M+T)\left\|K y_{0}\right\|$.

\section{Acknowledgement}

The work of the first author is supported by KOSEF.

\section{References}

[1] Balachandran, K. and Ilamaran, S., Existence and uniqueness of mild and strong solutions of a Volterra integrodifferential equation with nonlocal conditions, Tamkang J. Math. 28 (1997), 93-100.

[2] Bochenek, J., An abstract nonlinear second order differential equation, Annal. Polon. Math. 54 (1991), 155-166.

[3] Byszewski, L., Theorems about the existence and uniqueness of solutions of a semilinear evolution nonlocal Cauchy problem, J. Math. Anal. Appl. 162 (1991), 494505.

[4] Byszewski, L. and Acka, H., Existence of solutions of a semilinear functional differential evolution nonlocal problems, Nonl. Anal. Theory, Methods Appl. 34 (1998), 65-72.

[5] de Brito, E.H., A nonlinear hyperbolic equation, Inter. J. Math. Math. Sci. 3 (1980), 505-520.

[6] Levine, H.A., Instability and nonexistence of global solutions to nonlinear wave equations of the form $P u_{t t}=-A u+F(u)$, Trans. Amer. Math. Soc. 192 (1974), 121.

[7] Lin, Y. and Liu, J.H., Semilinear integrodifferential equations with nonlocal Cauchy problem, Nonl. Anal. Theory, Methods and Appl. 26 (1996), 1023-1033.

[8] Medeiros, L.A., On a new class of nonlinear wave equations, J. Math. Anal. Appl. 69 (1979), 252-262.

[9] Ntouyas, S.K. and Tsamatos, P.Ch., Global existence for second order functional semilinear equations, Period. Math. Hungar. 31 (1995), 223-228. 
[10] Ntouyas, S.K. and Tsamatos, P.Ch., Global existence for second order semilinear ordinary and delay integrodifferential equations with nonlocal conditions, Appl. Anal. 67 (1997), 245-257.

[11] Pereira, D.C., Existence, uniqueness and asymptotic behavior for solutions of the nonlinear beam equation, Nonl. Anal. Theory, Methods and Appl. 14 (1990), 613-623.

[12] Perla Menzala, G. and Zuazua, E., Timoshenko's plate equation as a singular limit of the dynamical von Karman system, J. Math. Pures Appl. 79 (2000), 73-94.

[13] Schaefer, H., Über die methode der a priori schranken, Math. Ann. 129 (1955), 415416.

[14] Timoshenko, S., Young, D.H. and Weaver, W., Vibration Problems in Engineering, John Wiley, New York 1974.

[15] Travis, C.C. and Webb, G.F., Compactness, regularity and uniform continuity properties of strongly continuous cosine families, Houston J. Math. 3 (1977), 555-567.

[16] Travis, C.C. and Webb, G.F., Cosine families and abstract nonlinear second order differential equations, Acta Math. Acad. Sci. Hungar. 32 (1978), 75-96. 


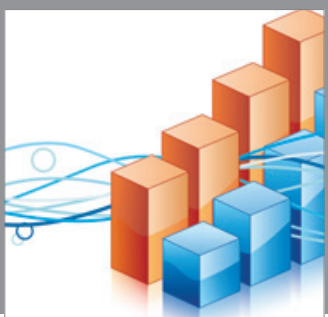

Advances in

Operations Research

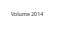

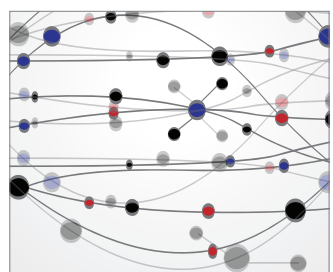

\section{The Scientific} World Journal
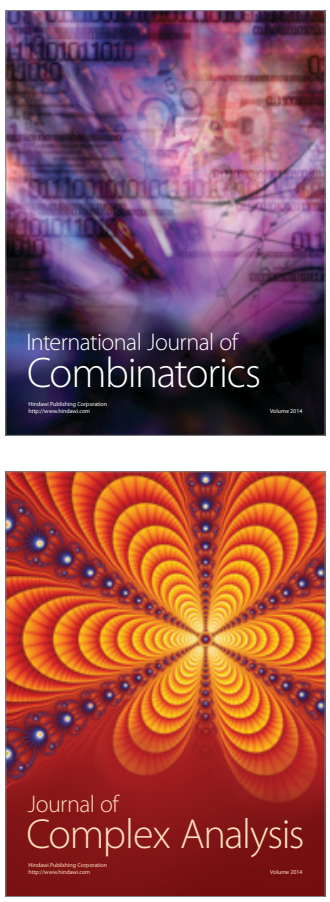

International Journal of

Mathematics and

Mathematical

Sciences
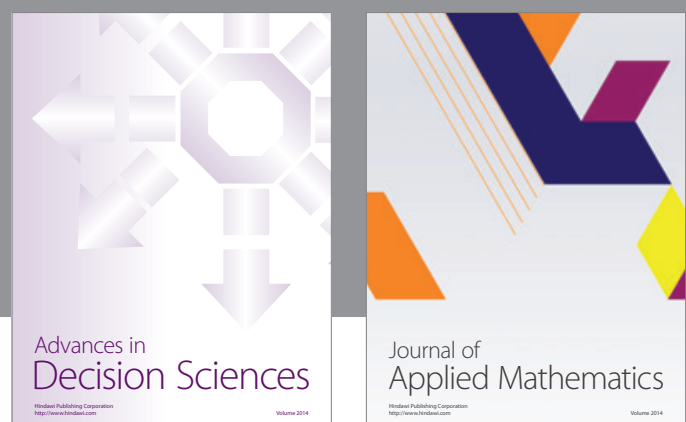

Journal of

Applied Mathematics
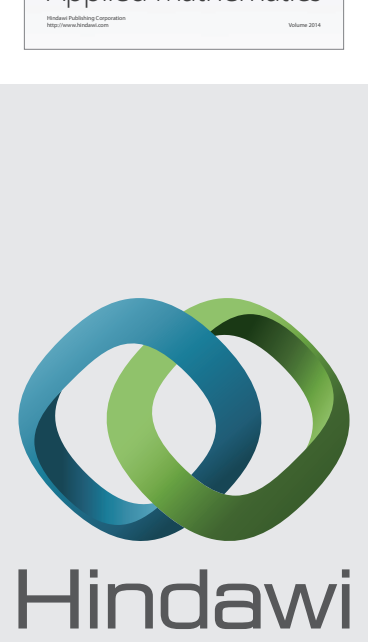

Submit your manuscripts at http://www.hindawi.com
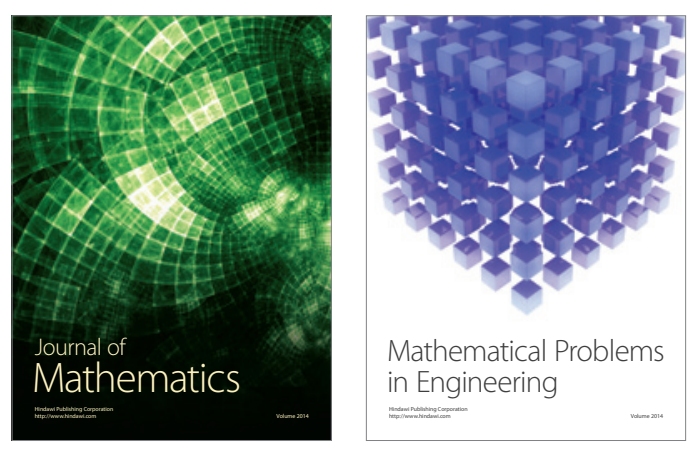

Mathematical Problems in Engineering
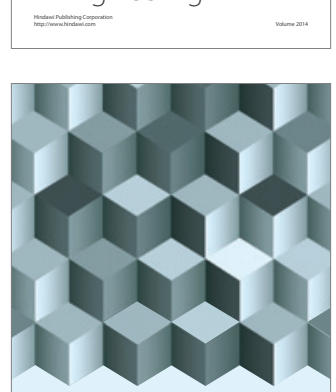

Journal of

Function Spaces
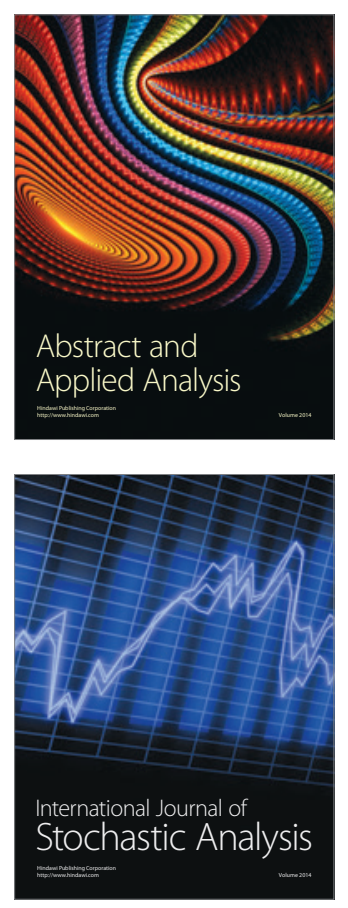

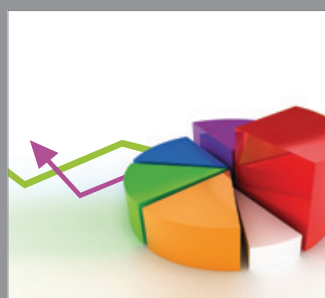

ournal of

Probability and Statistics

Promensencen
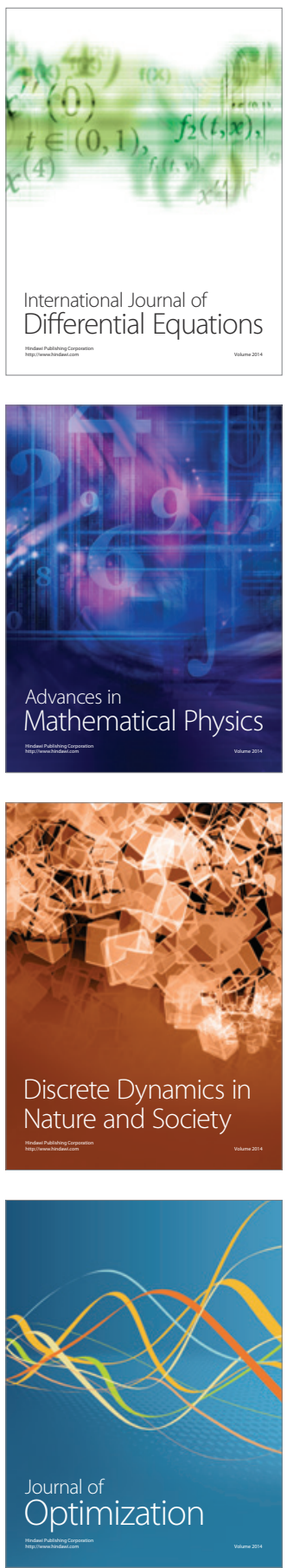